\title{
Dynamic Response of Suspended Underwater Systems
}

\author{
WILFRED D. IwaN \\ Division of Engineering and Applied Science, California Institude of Technology, Pasadena, California 91109
}

\begin{abstract}
A method is presented for analyzing the steady-state or stationary random heave response of suspended underwater systems. The nonlinear continuous system is replaced by a discretized pseudolinear system by means of an equation difference minimization technique. The resulting pseudolinear system is solved by an iterative scheme. An example of the application of the proposed method of analysis to a suspended hydrophone system is given. The example shows that the nonlinear pressure drag forces have a very significant effect on the over-all response of the system. The nature of the response is discussed and some practical conclusions are given.
\end{abstract}

\section{INTRODUCTION}

Dynamic heave response is an important consideration in a variety of suspended underwater structures, including deep-sea drilling systems, recovery systems, and acoustical detection systems. There is some experimental data on the heave response of certain specific structures, ${ }^{1}$ but in general the available data is quite limited. In the absence of experimental data for a given system configuration, the response must be estimated on the basis of theoretical analysis. The techniques used in such an analysis might be exact or approximate but should treat the problem in a manner which is both physically and mathematically consistent.

If all system nonlinearities are neglected, the analysis of a suspended underwater system becomes a very straightforward linear problem. There are many techniques available for solving this type of problem. When significant system nonlinearities such as hydrodynamic pressure drag forces are included, the theoretical problem becomes much more formidable. There is relatively little published work in this area, especially in the open literature. Several investigators ${ }^{\mathbf{2}}{ }^{3}$ have used numerical integration techniques to solve particular transient response problems. However, such techniques are not well suited to analysis of the steadystate or random response problems. The latter problem is particularly important in cases where the structure is excited by wind-induced ocean waves.

The most widely referenced theoretical treatment of the steady-state problem is that of Germeles contained in a 1963 report by the Arthur D. Little Co. ${ }^{4}$ The analysis of numerous specific systems ${ }^{1,5}$ has been based on the approximate analysis presented in this report. Unfortunately, this analysis does not appear to deal adequately with the nonlinearities of the problem, for it predicts that the system response is linearly proportional to the system excitation. This is not generally true. In addition, the approach presented cannot correctly be applied to the random response problem even though such application has been attempted.

This study discusses an approximate analytic approach to the heave response problem which is applicable for both steady-state harmonic and random excitation. The approach is based on the concept of finding a substitute linear system to replace the actual nonlinear system in such a way that the mean-square difference between the two systems is minimized. The effective linear system is uniquely determined and can be treated by means of a relatively simple transfer function analysis for the subject class of systems.

\section{FORMULATION}

Consider the suspended underwater system shown schematically in Fig. 1(a). For the purposes of analysis, this system may be replaced by the lumped parameter system shown in Fig. 1(b). The first 4-5 modes of the system will generally be quite accurately represented if $N>50$, where $N$ is the number of discrete elements used to approximate the continuous member. In the discretized analog, the buoy has been replaced by an equivalent spring $K_{b}$ and "effective" mass ${ }^{6} M_{b}$, where

$$
K_{b}=\rho g A_{b},
$$


in which $\rho$ is the density of the surrounding water, $g$ is the acceleration of gravity, and $A_{b}$ is the effective area of the buoy for buoyancy calculations. Let the cable density, area, and effective elastic modulus be $\mu_{c}, A_{c}$, and $E_{c}$, respectively. Then,

$$
\begin{aligned}
& k_{c}=N K_{c}=A_{c} E_{c} N / L, \\
& m_{c}=M_{c} / N=\mu_{c} A_{c} L / N .
\end{aligned}
$$

The effective mass of the payload will be denoted by $M_{p}$.

Each of the elements in the discretized system of Fig. 1(b) is assumed to be subject to a hydrodynamic drag force which is a function of the velocity of the element. In the case of the buoy, it is assumed that the drag force is a function of the relative velocity of the buoy and sea. In all other cases, the drag force is assumed to be a function of the absolute velocity of the element. The hydrodynamic drag force on the buoy will be denoted by $h_{b}\left(\dot{y}_{b}-\dot{y}_{s}\right)$, on the $i$ th element of the cable by $h_{j}\left(\dot{y}_{j}\right)$ and on the payload by $h_{p}\left(\dot{y}_{p}\right)$.

As a consequence of the above assumptions, the differential equations of motion of the system may be written as

$$
\begin{aligned}
M_{b} \ddot{y}_{b}+h_{b}\left(\dot{y}_{b}-\dot{y}_{s}\right)+k_{b}\left(y_{b}-y_{s}\right)+2 k_{c}\left(y_{b}-y_{1}\right) & =0, \\
m_{c} \ddot{y}_{1}+h_{1}\left(\dot{y}_{1}\right)+k_{c}\left(3 y_{1}-y_{2}-2 y_{b}\right) & =0, \\
: & \\
m_{c} \ddot{y}_{j}+h_{j}\left(\dot{y}_{j}\right)+k_{c}\left(2 y_{j}-y_{j+1}-y_{j-1}\right) & =0, \\
\vdots & \\
m_{c} \ddot{y}_{N}+h_{N}\left(\dot{y}_{N}\right)+k_{o}\left(3 y_{N}-y_{N-1}-2 y_{p}\right) & =0 \\
M_{p} \ddot{y}_{p}+h_{p}\left(\dot{y}_{p}\right)+2 k_{c}\left(y_{p}-y_{N}\right) & =0 .
\end{aligned}
$$

$y_{8}=y_{s}(t)$ is assumed to be a prescribed sea-wave excitation which may be deterministic or random.

\section{METHOD OF ANALYSIS}

Let the system of Eqs. 3 be denoted by

$$
\mathbf{D}_{\mathbf{1}}(\mathbf{y}, t)=\mathbf{0}
$$

where $\mathbf{y}$ is the $N+2$ vector whose components are the dependent variables $y_{b}, y_{1}, y_{2}, \cdots, y_{N}, y_{p}$. As a means of generating an approximate solution of Eq. 4, consider an auxiliary set of linear equations

$$
\mathbf{D}_{2}(\mathbf{y}, t)=\mathbf{0}
$$

where this set of equations has the specific form

$$
\begin{aligned}
M_{b} \ddot{y}_{b}+c_{b}\left(\dot{y}_{b}-\dot{y}_{\theta}\right)+k_{b}\left(y_{b}-y_{s}\right)+2 k_{c}\left(y_{b}-y_{1}\right) & =0, \\
m_{c} \ddot{y}_{1}+c_{1} \dot{y}_{1}+k_{c}\left(3 y_{1}-y_{2}-2 y_{b}\right) & =0, \\
: & \\
m_{c} \ddot{y}_{j}+c_{j} \dot{y}_{j}+k_{c}\left(2 y_{j}-y_{j+1}-y_{j-1}\right) & =0 \\
: & \\
m_{c} \ddot{y}_{N}+c_{N} \dot{y}_{N}+k_{c}\left(3 y_{N}-y_{N-1}-2 y_{p}\right) & =0 \\
M_{p} \ddot{y}_{p}+c_{p} \dot{y}_{p}+2 k_{c}\left(y_{p}-y_{N}\right) & =0 .
\end{aligned}
$$

The solution of this linear set of equations may be determined in a straightforward manner and will be a

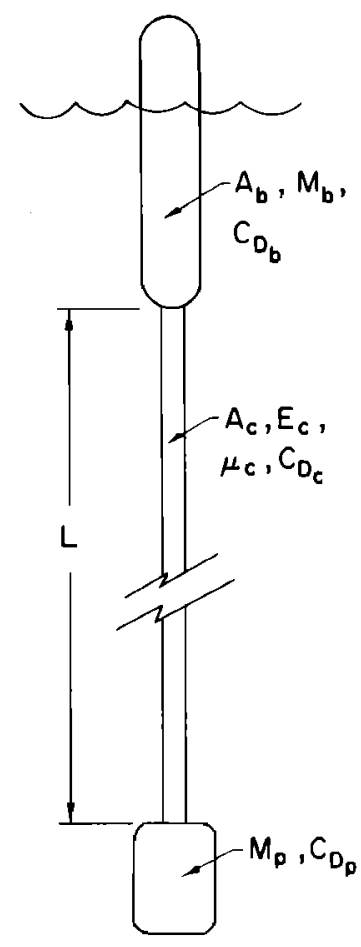

(a)

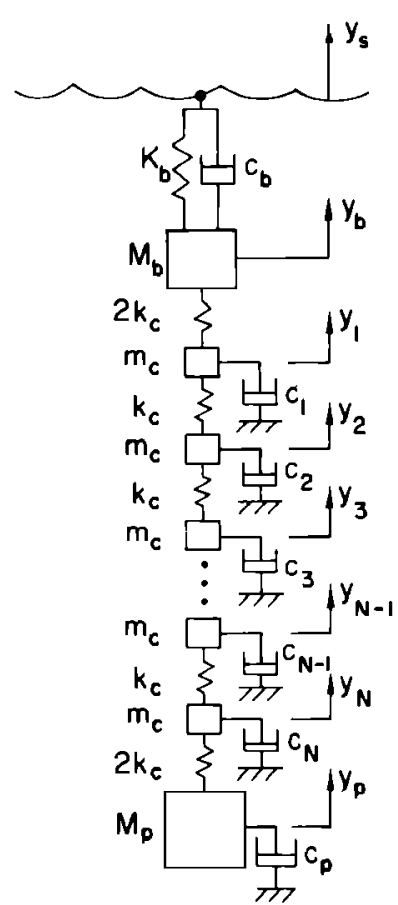

(b)
FIG. 1. Suspended underwater system. (a) Physical system. (b) Discretized model.

member of some class of solutions e. The particular member of this class of solutions is determined by specification of the parameters $c_{b}, c_{j}$, and $c_{p}$. The method of approximation is to select the linear system parameters $c_{b}, c_{j}$, and $c_{p}$ so that the mean value of the square of the Euclidean norm of the difference between the two systems of Eqs. 4 and 5 is a minimum for all members of the class of solutions $\mathrm{C}$. Hence,

$$
\text { mean }\left[\varepsilon^{\prime} \varepsilon\right]=\text { minimum w.r.t. } c_{b}, c_{j}, c_{p} \forall \mathbf{y} \in \mathbb{e} .
$$

The prime denotes the transpose operation and $\varepsilon$ is defined as

$$
\varepsilon \equiv \mathbf{D}_{2}(\mathbf{y}, t)-\mathbf{D}_{1}(\mathbf{y}, t) \text {. }
$$

The value of $\mathbf{y}$ to be used in Eq. 8 is the solution of Eqs. 6. The mean value in the minimization criterion Eq. 7 will be interpreted differently, depending on whether the system is deterninistic or nondeterministic.

It may be shown (see Ref. 7) that in general the parameters $c_{b}, c_{j}$, and $c_{p}$ which satisfy the equation difference minimization criterion are unique and may be written as

$$
\begin{aligned}
c_{b} & =\operatorname{mean}\left[\left(\dot{y}_{b}-\dot{y}_{s}\right) h_{b}\left(\dot{y}_{b}-\dot{y}_{s}\right)\right] / \operatorname{mean}\left[\left(\dot{y}_{b}-\dot{y}_{\theta}\right)^{2}\right], \\
c_{j} & =\operatorname{mean}\left[\dot{y}_{j} h_{j}\left(\dot{y}_{j}\right)\right] / \operatorname{mean}\left[\dot{y}_{j}{ }^{2}\right], \quad j=1, \cdots, N, \\
c_{p} & =\operatorname{mean}\left[\dot{y}_{p} h_{p}\left(\dot{y}_{p}\right)\right] / \operatorname{mean}\left[\dot{y}_{p}{ }^{2}\right] .
\end{aligned}
$$


With these parameters specified, a particular member of the class of solutions $\mathfrak{C}$ is identified, and the approximate solution of Eqs. 3 is thus determined. The form of this solution and the type of information which it will yield depend in general upon the particular nature of the problem considered. For a steady-state deterministic excitation and response, the solution will be harmonic and the amplitude and phase of the response are normally sought. For a stationary random excitation and response, only probabilistic information may be determined and this normally means determination of the mean-square response amplitude or response power spectrum.

The accuracy of the generalized equivalent linear system approach has been examined for simple deterministic nonlinear systems ${ }^{\mathrm{a}}$ and for certain multidegree-of-freedom stochastically excited nonlinear systems. ${ }^{9}$ In general, the results obtained are found to be well within the range of acceptable engineering accuracy.

For deterministic excitation, the present approach gives essentially the same result as the Ritz-Galerkin method, the method of energy balance, or the method of slowly varying parameters. These methods and variations have been used by some investigators to study related systems. For random excitation, the present approach might be thought of as an extension of the method of equivalent linearization. There do not appear to be any instances in the open literature where such an approach has been applied to the analysis of suspended underwater systems. A similar approach has, however, been used in the analysis of offshore drilling towers. ${ }^{10}$

Since the parameters $c_{b}, c_{j}, c_{p}$ depend upon the solution itself, an iterative procedure will generally be required to solve the system of Eq. 6 . However, owing to the chain-like form of the subject system, a solution may be obtained relatively simply for either the deterministic or random problem.

\section{SOLUTION TECHNIQUE}

The most direct method of obtaining a solution of the system of Eqs. 6 is to generate the complex transfer function of the system. This may be accomplished as follows : Let

$$
\begin{aligned}
y_{n}=Y_{a} e^{i \omega t} ; \quad y_{b} & =Y_{b} e^{i \omega t} ; \quad y_{p}=Y_{p} e^{i \omega t} ; \\
y_{j} & =Y_{j} e^{i \omega t},
\end{aligned}
$$

where the $Y$ 's will in general be complex. Then, from Eq. 6, it may be shown that the transfer function $H_{p s}(i \omega)$ can be written as

$$
H_{p s}(i \omega) \equiv \frac{Y_{p}}{Y_{a}}=\frac{1+i \omega C_{b} / k_{b}}{F_{s}+i G_{a}},
$$

where

$$
\begin{aligned}
F_{b} & =\left[\left(K_{b}+2 k_{c}-\omega^{2} M_{b}\right) F_{b}-\omega c_{b} G_{b}-2 k_{c} F_{1}\right] / K_{b}, \\
G_{b} & =\left[\left(K_{b}+2 k_{c}-\omega^{2} M_{b}\right) G_{b}+\omega c_{b} F_{b}-2 k_{c} G_{1}\right] / K_{b}, \\
F_{b} & =\left[\left(3 k_{c}-\omega^{2} m_{c}\right) F_{1}-\omega c_{1} G_{1}-k_{c} F_{2}\right] / 2 k_{c}, \\
G_{b} & =\left[\left(3 k_{c}-\omega^{2} m_{c}\right) G_{1}+\omega c_{1} F_{1}-k_{c} G_{2}\right] / 2 k_{c}, \\
F_{j-1} & =\left[\left(2 k_{c}-\omega^{2} m_{c}\right) F_{2}-\omega c_{j} G_{j}-k_{c} F_{j+1}\right] / k_{c}, \\
j=2, \cdots, N-1, & j, j=2, \cdots, N-1, \\
G_{j-1} & =\left[\left(2 k_{c}-\omega^{2} m_{c}\right) G_{j}+\omega c_{j} F_{j}-k_{c} G_{j+1}\right] / k_{c}, \\
j=2, & \\
F_{N-1} & =\left[\left(3 k_{c}-\omega^{2} m_{c}\right) F_{N}-\omega c_{N} G_{N}-2 k_{c}\right] / k_{c}, \\
G_{N-1} & =\left[\left(3 k_{c}-\omega^{2} m_{c}\right) G_{N}+\omega c_{N} F_{N}\right] / k_{c}, \\
F_{N} & =\left(1-\omega^{2} M_{p} / 2 k_{c}\right), \\
G_{N} & =\omega c_{p} / 2 k_{c} .
\end{aligned}
$$

It will be noted that Eqs. 12 can readily be solved numerically in reverse order without any computational difficulty once the parameters $c_{b}, c_{j}$, and $c_{p}$ are specified.

The transfer function between $Y_{\mathrm{a}}$ and any other element of the system may be expressed in a form similar to Eq. 11. Hence

$$
\begin{aligned}
& H_{j a}(i \omega) \equiv Y_{j} / Y_{s}=\left(F_{j}+i G_{j}\right) H_{p s}(i \omega), \\
& H_{b s}(i \omega)=Y_{b} / Y_{c}=\left(F_{b}+i G_{b}\right) H_{p s}(i \omega) .
\end{aligned}
$$

Once the complex transfer functions of the system are known, the steady-state harmonic or stationary random response of the system may be determined by standard techniques. Note, however, that the factors $F_{b}, F_{j}, F_{p}, G_{b}, G_{i}$, and $G_{p}$ in Eqs. 12 will normally be functions of the solution itself.

\section{A. Steady-State Harmonic Response}

For steady-state harmonic response, the dependent variables $y_{s}, y_{b}, y_{j}$, and $y_{p}$ will be trigonometric and the mean values of Eq. 9 will have the form

$\operatorname{mean}[\dot{z h}(\bar{z})]=\frac{\omega|Z|}{2 \pi} \int_{0}^{2 \pi} h(-\omega|Z| \sin \theta) \sin \theta d \theta$

In Eq. $14,|Z|$ is the amplitude of the variable $z$ which may represent any one of the dependent variables of the system or combination thereof. Hence, for example, it is seen that

$$
c_{p}=\frac{1}{\pi \omega\left|Y_{p}\right|} \int_{0}^{2 \pi} h_{p}\left(-\omega\left|Y_{p}\right| \sin \theta\right) \sin \theta d \theta
$$

Analogous expressions would be obtained for the other effective linear damping coefficients.

For dissipative forces that arise solely from hydrodynamic pressure drag, the functions $h(\dot{z})$ will have the form

$$
h(\dot{z})=\left(C_{d} \rho A / 2\right) \dot{z}|\dot{z}|,
$$


where $C_{d}$ is the drag coefficient of the particular element and $A$ is the effective area of the element for drag calculations. In this case it is easily shown that, for example,

$$
c_{p}=\left(\frac{8}{3 \pi}\right)\left(\frac{C_{d_{p} \rho} A_{p}}{2}\right) \omega\left|Y_{p}\right|
$$

Analogous expressions will be obtained for $c_{b}$ and $c_{j}$, except that they will involve the drag and area parameters appropriate to the particular element.

With the effective viscous damping coefficients $c_{b}, c_{j}$, and $c_{p}$ thus determined, the amplitude of the steadystate response of the payload may be deduced from the relation

$$
\left|Y_{p}\right|=\left|H_{p s}(i \omega)\right|\left|Y_{s}\right| \text {. }
$$

\section{B. Stationary Random Response}

For stationary random response, only statistical information will be available concerning the variables $y_{a}, y_{b}$, etc. Therefore, the means in the relations of Eqs. 9 must be interpreted in a statistical sense. Let these means denote the mathematical expectation or ensemble average. Then they may be written as

$$
\operatorname{mean}[\dot{z} h(\dot{z})]=E[\dot{z} h(\dot{z})] \text {, }
$$

where $z$ denotes any one of the dependent response variables of the system or combinations therefore. If the excitation process $y_{8}(t)$ is Gaussian distributed, the response processes associated with the auxiliary linear system will also be Gaussian distributed and

$$
E[\dot{z} h(\dot{z})]=\frac{1}{\sigma_{\dot{z}}(2 \pi)} \int_{-\infty}^{\infty} \dot{z} h(\dot{z}) \exp \left(-\frac{\dot{z}^{2}}{2 \sigma_{\dot{z}}^{2}}\right) d \dot{z},
$$

where $\sigma_{\dot{z}}$ denotes the root-mean-square ( $\mathrm{mms}$ ) value of the process $z(t)$. Hence, for example,

$$
c_{p}=\frac{1}{\sigma_{\dot{y}_{p}}{ }^{3}(2 \pi)^{1}} \int_{-\infty}^{\infty} \dot{y}_{p} h_{p}\left(\dot{y}_{p}\right) \exp \left(-\frac{\dot{y}_{p}^{2}}{2 \sigma_{\dot{y}_{p}}{ }^{2}}\right) d \dot{y}_{p} .
$$

For dissipative pressure drag forces of the form of (Eq. 16), this gives

$$
c_{p}=\left(\frac{8}{\pi}\right)^{3}\left(\frac{C_{d_{p} \rho_{p}} A_{p}}{2}\right) \sigma_{\dot{y}_{p}} .
$$

Analogous expressions may be obtained for $c_{b}$ and $c_{j}$.

The result of Eq. 22 clearly differs from that obtained for the steady-state harmonic response, Eq. 17. For the random response problem, the parameters of the effective linear system must depend upon the statistical properties of the response ( $\sigma_{\dot{\nu}_{p}}$ here). The results of the harmonic analysis cannot be applied in this case, for they involve the explicit assumption that the response is harmonic with a single specified fre- quency. This assumption is not valid for the stationary random problem. The response analysis presented in Ref. 4 uses the harmonic assumption and therefore cannot correctly be applied to the random problem.

With the effective viscous damping coefficients $c_{b}$, $c_{j}$, and $c_{p}$ determined, the rms response of the payload may be obtained by means of a spectral density transformation and integration of the form

$$
{\sigma_{i_{p}}}^{2}=\int_{-\infty}^{\infty}\left|H_{p s}(i \omega)\right|^{2} \Phi_{s s}(\omega) d \omega,
$$

where $\Phi_{s s}(\omega)$ is the spectral density of the sea-wave excitation. Since $H_{p s}(i \omega)$ is a function of the rms velocity responses $\sigma_{y_{p}}, \sigma_{u_{b}}$, etc., it will be necessary to employ an iterative solution procedure and also to make use of relations of the form

$$
\sigma_{\dot{y}_{p}}{ }^{2}=\int_{-\infty}^{\infty} \omega^{2}\left|H_{p q}(i \omega)\right|^{2} \Phi_{z s}(\omega) d \omega .
$$

\section{APPLICATION}

As an example of the application of the present technique of analysis, consider the problem of determining the heave response of a suspended hydrophone array. In order to simplify the analysis but still retain the basic character of the real problem, it will be assumed that the primary source of pressure drag damping is the hydrophone or payload itself; that is, it will be assumed that $c_{b}=c_{j}=0 ; j=1, \cdots, N$. For this particular case, the response will be a function of the following system parameters:

$$
\begin{aligned}
& \delta_{c} \equiv M_{c} / M_{p}, \\
& \delta_{b} \equiv M_{b} / M_{p}, \\
& f_{p} \equiv(1 / 2 \pi)\left(K_{c} / M_{p}\right)^{\sharp}, \\
& f_{b} \equiv(1 / 2 \pi)\left[K_{b} /\left(M_{b}+M_{p}+M_{c}\right)\right]^{\ddagger}, \\
& l^{*} \equiv 2 M_{p} / C_{d_{p} \rho} \rho A_{p} .
\end{aligned}
$$

The physical significance of $\delta_{c}$ and $\delta_{b}$ is clear from their definitions. $f_{p}$ is the natural frequency of the cablehydrophone system when $\delta_{c}=0$, and $f_{b}$ is the natural frequency of the buoy-cable-hydrophone system when $K_{c} \rightarrow \infty$. The parameter $l^{*}$ has the dimensions of a length and will be referred to as the characteristic drag length of the hydrophone array. This parameter specifies the magnitude of the pressure drag force on the hydrophone array and is primarily a function of the geometry of this element. A flat plate like structure would have a very low characteristic drag length while a long slender structure would have a much larger $l^{*}$.

\section{A. Harmonic Excitation}

Valuable insight into the dynamic character of the suspended system may be obtained from an analysis of its steady-state harmonic response. For such an 
analysis, a constant amplitude single-frequency wave motion is applied to the buoy and the effective linear damping coefficient is determined from Eq. 17. Let

$$
y_{s}=\bar{Y}_{s} \cos 2 \pi f_{s} t, \quad y_{p}=\bar{Y}_{p} \cos \left(2 \pi f_{s} t-\varphi\right),
$$

where $f_{0}$ is the wave excitation frequency and $\bar{Y}_{8}$ the zero-to-peak wave amplitude. Then, the amplitude of the transfer function for the system becomes

$$
\left|H_{p s}(i \omega)\right|=\bar{Y}_{p} / \bar{Y}_{s} ; \omega=2 \pi f_{o} .
$$

In Fig. 2, this transfer function amplitude is plotted as a function of excitation frequency $f$, for the system $f_{p}=0.2 \mathrm{~Hz}, \delta_{c}=0.2, \delta_{b}=2.0, l^{*}=10 \mathrm{ft}$, and a range of values of buoy natural frequency. The excitation amplitude $\bar{Y}$, is equal to $1.0 \mathrm{ft}$.

Consider the results of the system in which $f_{b} \rightarrow \infty$; an extremely flat, stiff buoy. In this case, a rather interesting result is observed, namely, that the peak value of the transfer function is very nearly a constant for each of the modes of the system. This implies that the effective damping in the higher modes of the drag damped system is substantially greater than it would be in a purely viscous damped system.

To demonstrate this point, Fig. 3 shows the peak value of $\bar{Y}_{p} / \bar{Y}_{s}$ in each of the first four modes of the system for the postulated drag damping and also for a viscous damping selected to give an identical first mode peak response. This viscous damping is obtained by conceptually attaching a linear dashpot from the payload to ground. Results are given for $\bar{Y}_{d}=0.5,1.0$, and $2.0 \mathrm{ft}$. Clearly, the use of viscous damping in modeling such a system would lead to a very great overestimation of the maximum heave response in the higher modes. Conversely, if the higher modes were more accurately represented with viscous damping, the first mode response would be substantially underestimated. Hence, it is important that the full nonlinear analysis be employed when more than one mode is involved in the response.

Figure 3 clearly indicates the nonlinear nature of the system in that the transfer ratio $\bar{Y}_{p} / \bar{Y}_{s}$ is seen to be a function of the excitation amplitude $\bar{Y}_{g}$. This stands in contrast to the results of Ref. 4, which gives $\bar{Y}_{p} / \bar{Y}_{\text {a }}$ as independent of $\bar{Y}_{g}$. Note that $\bar{Y}_{p} / \bar{Y}_{\text {s }}$ actually decreases as $\bar{Y}_{s}$ increases for the drag damped system. Hence, this form of damping tends to be more effective than viscous damping at high amplitudes of response and less effective at low amplitudes.

Figure 2 also indicates the manner in which the steady-state response changes as the buoy frequency decreases; i.e., the buoy becomes more "spar"-like. As expected, a finite buoy frequency introduces a highfrequency roll off which suppresses most of the higher mode peaks. Note, however, that the over-all peak response of the system with a finite buoy frequency is greater than that for the system with $f_{b} \rightarrow \infty$ for all

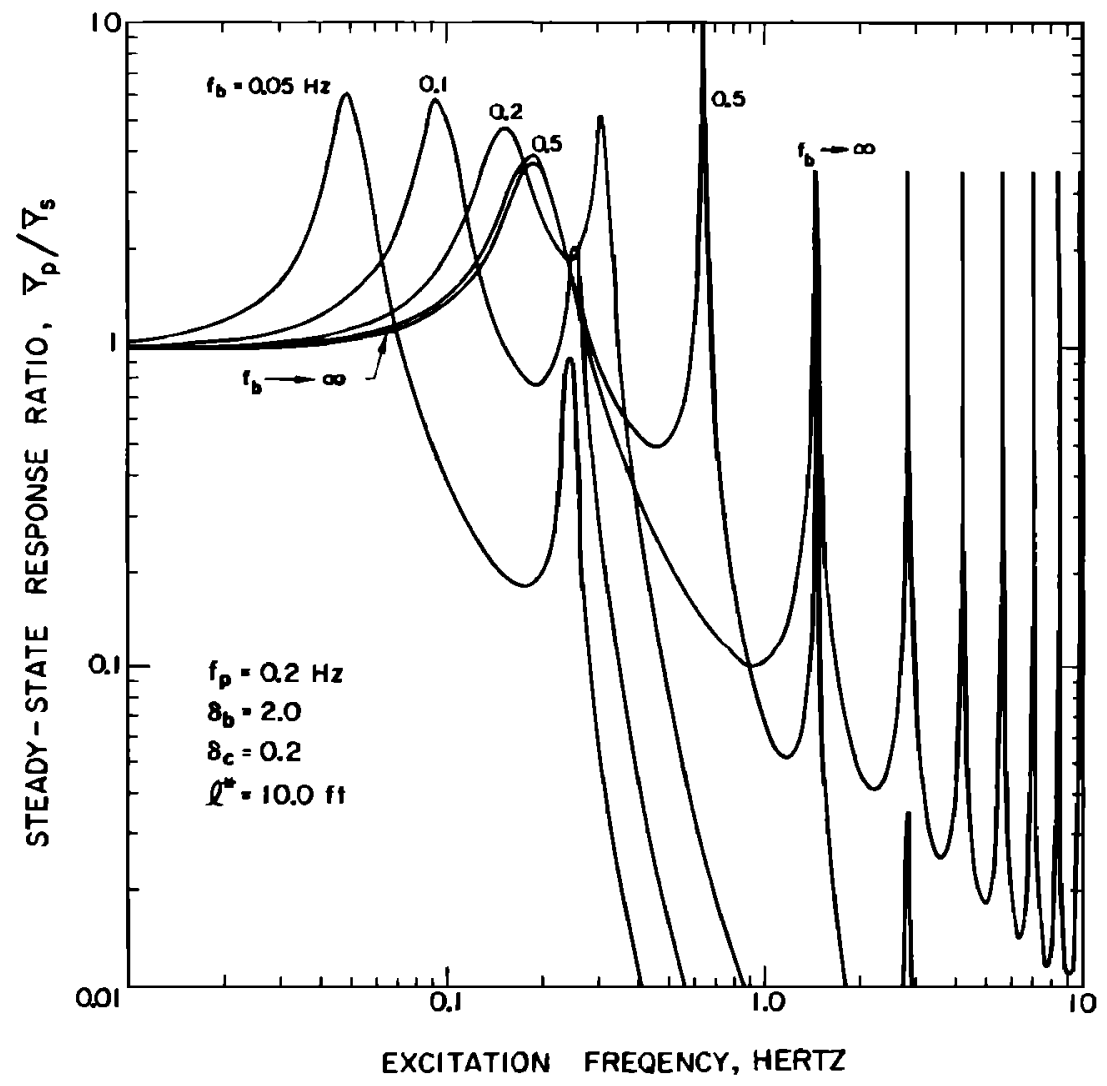

FiG. 2. Steady-state response ratio versus excitation frequency; harmonic excitation. 
FIG. 3. Maximum steady-state response ratio, $\vec{Y}_{p} / \bar{Y}_{a}$, versus mode number for drag damping and viscous damping selected to give same first mode response.

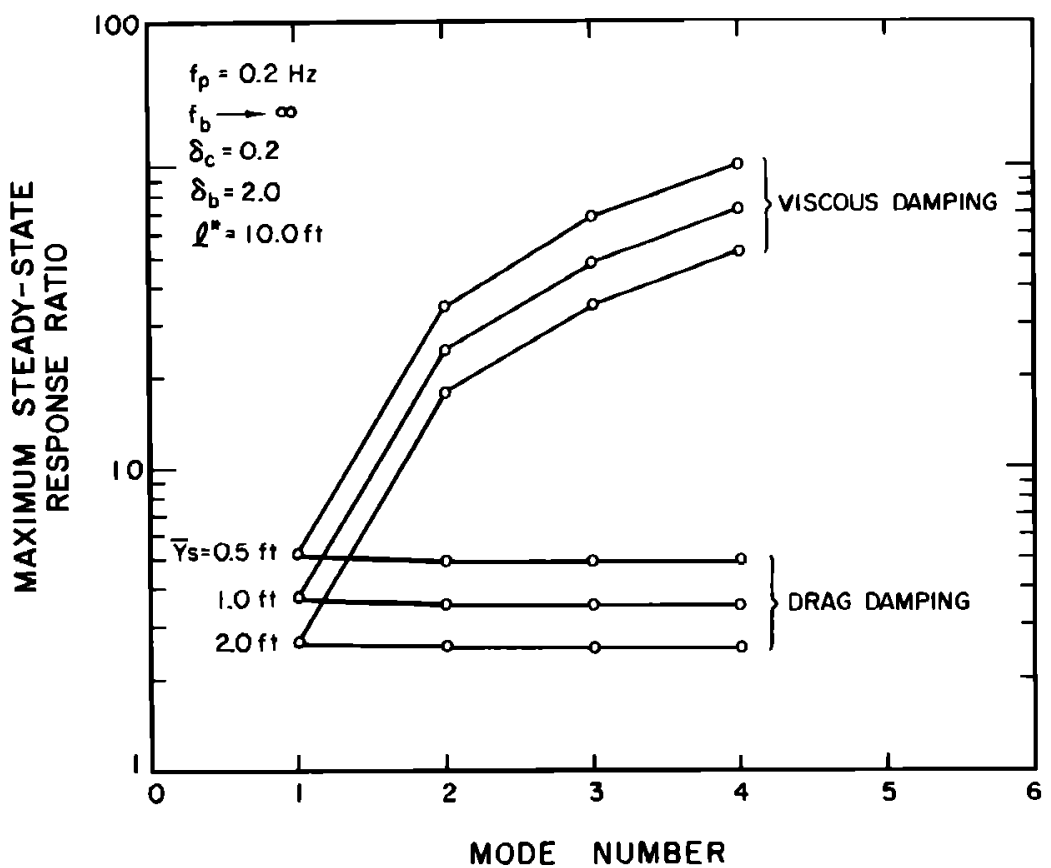

of the cases examined. The effectiveness of the buoy as an isolator will therefore depend strongly on the frequency content of the excitation. This point is reinforced by the results of the next section.

\section{B. Random Sea State}

For ocean waves, it is well known that wave amplitude or energy is a strong function of wave frequency or wavelength. One mathematical model for this functional dependence is provided by the PiersonMoskowitz spectral density function for fully developed waves in a random sea. ${ }^{11}$ This spectral density has been inferred from experimental data and may be written in the form

$$
\begin{aligned}
\Phi_{a a}(\omega)=8.10 \times 10^{-3} g^{2} \omega^{-5} & \\
& \times \exp \left(-1.62 \times 10^{-2} g^{2} / H_{t}^{2} \omega^{4}\right),
\end{aligned}
$$

where $H_{\mathbf{l}}$ is the most significant wave height. $H_{\mathbf{j}}$ is twice the rms value of the excitation; that is

$$
H_{1}=2\left[2 \int_{0}^{\infty} \Phi_{s s}(\omega) d \omega\right]^{1} .
$$

The Pierson-Moskowitz sea-wave spectrum is shown in Fig. 4. It is noted that the peak of the spectrum moves progressively toward lower frequency as the wave amplitude increases. The effects of this frequency shift on the response of the hydrophone assembly will be seen later. In addition to the assumed form for the spectral density of the wave input, it will be assumed that the wave motion is Gaussian distributed.

The response of the suspended hydrophone assembly may be determined by using the effective linear damping coefficient defined by E.q 22, and the spectral density of $\mathrm{Eq}$. 28. The functional dependence of the rms heave response of the payload $\sigma_{y_{p}}$ on the various system

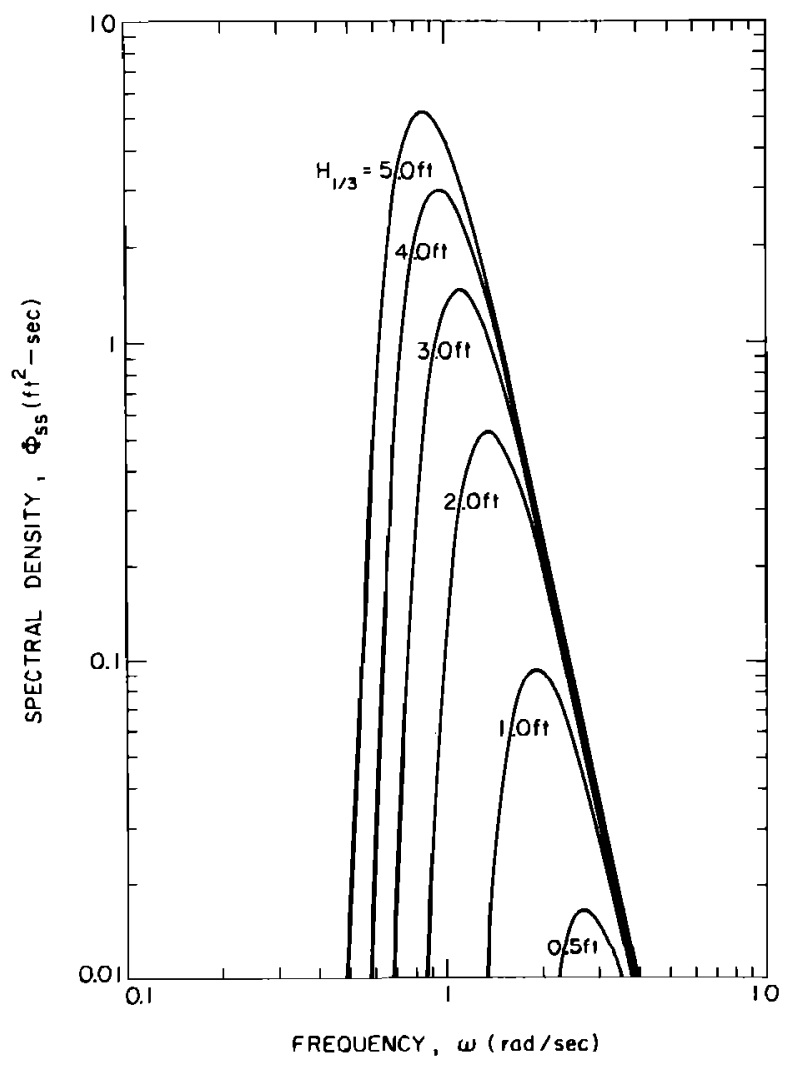

Fig. 4. Pierson-Moskowitz sea-wave spectrum. 


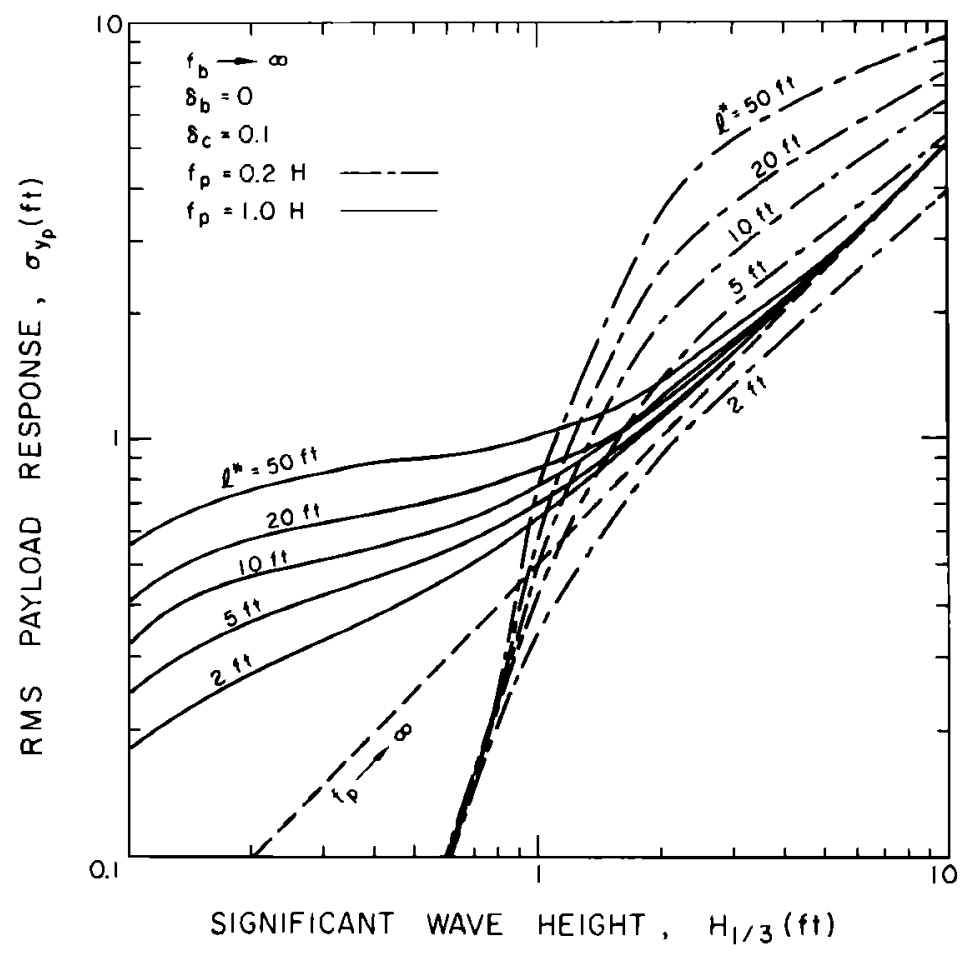

FIG. 5. rms payload response versus significant wave height.

parameters and the excitation parameter $H_{\mathfrak{l}}$ is shown in Figs. 5-7.

Figure 5 shows the manner in which the response depends upon the characteristic drag length $l^{*}$ of the stiff buoy $\left(f_{b} \rightarrow \infty\right)$ hydrophone system for two different values of cable-hydrophone frequency $f_{p}$. In all but one of the cases shown, the rms response amplitude $\sigma_{y_{p}}$ exceeds the excitation rms amplitude for some range of values of significant wave height $H_{\mathfrak{b}}$. The dashed line represents the locus of equal response and excitation rms values. For the two systems shown, the stiffer hydrophone suspension $\left(f_{p}=1.0 \mathrm{~Hz}\right)$ exhibits a lower level of response for values of $H_{t}$ greater than approximately $1.0 \mathrm{ft}$ while the more flexible suspension $\left(f_{p}=0.2 \mathrm{~Hz}\right)$ performs better for lower values of $H_{3}$.

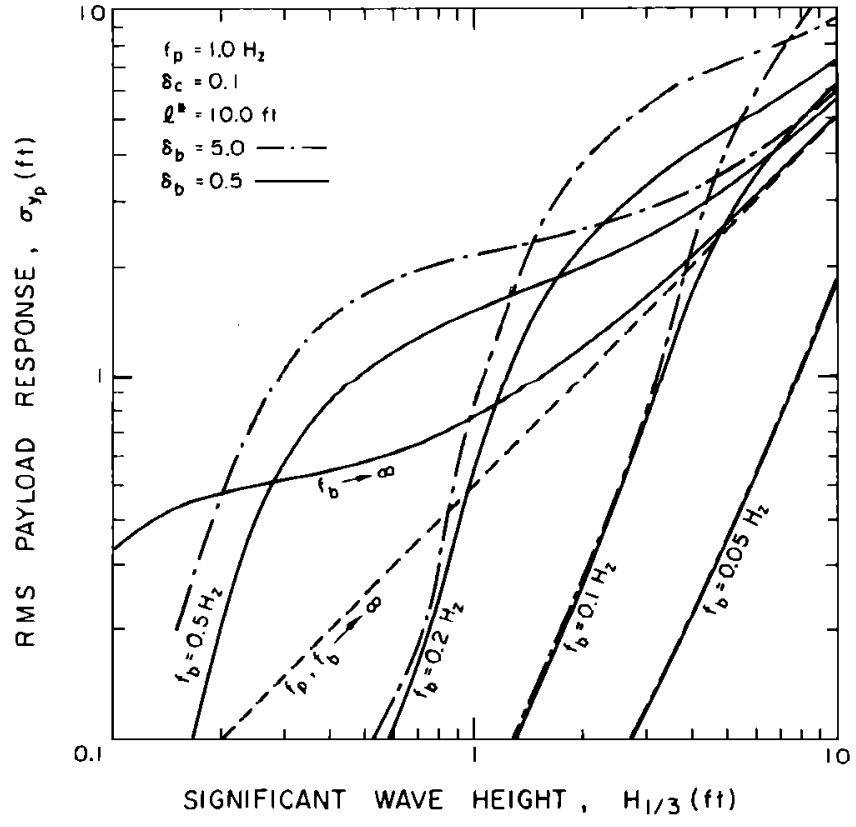

Fig. 6. rms payload response versus significant wave height. 
Fig. 7. rms payload response versus significant wave height.

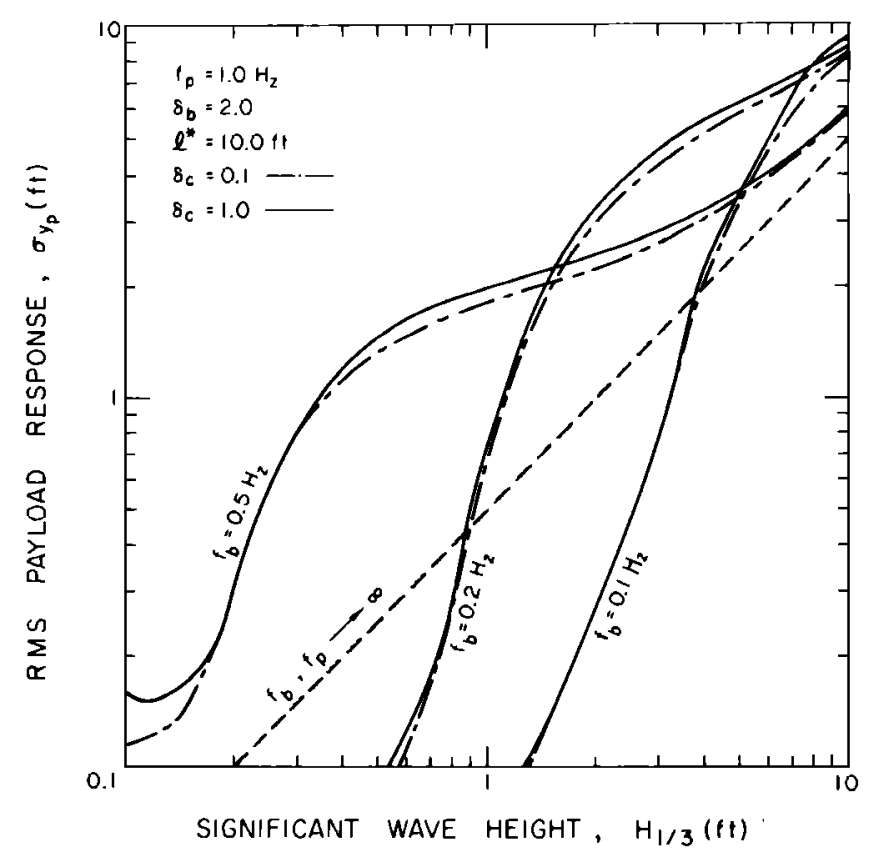

system is replaced by a fully discretized pseudolinear system using an equation difference minimization technique. As a consequence of the chain-like character of the simple suspended system, the effective transfer function for any point in the system is easily determined. The resulting pseudolinear system may be solved by an iterative scheme.

The proposed analysis technique has been employed in a parameter study of a suspended hydrophone assembly. The example indicates that the nonlinearities introduced by pressure drag forces have a significant effect on the response of the system. For a random sea, the hydrophone response is most strongly a function of the characteristic drag length $l^{*}$ (damping) of the array and the buoy natural frequency. Cable and mass ratios appear to have a secondary affect on the over-all response of the system.

\section{ACKNOWLEDGMENT}

This investigation was sponsored in part by a grant

from the National Science Foundation.

Figure 7 shows how the response depends upon the cable mass ratio $\delta_{c}$. For the systems considered, it is concluded that the response is relatively insensitive to this particular parameter. This is most likely a consequence of the range of values of $\delta_{c}$ considered and the high effective damping of the higher modes of the cable system as discussed earlier in connection with the steady-state response.

\section{SUMMARY AND CONCLUSIONS}

An approximate method for determining the steadystate or stationary random heave response of suspended underwater systems has been presented. In the proposed approach, the nonlinear discrete-continuous
1 B. J. Muga, "Mechanics of Raising and Lowering Heavy Loads in the Ocean: Experimental Results," U. S. Naval Civil Eng. Lab. Tech. Rep. R-543 (Sept. 1967).

2 T. S. Walton and H. Polacheck "Calculation of Transient Motion of Submerged Cables," Math. Comp. 14, 27-46 (1966).

a P. A. Laura and J. E. Goeller, "On the Dynamic Behavior of a Cable System in a Recovery Operation," J. Acoust. Soc. Amer. 49, 615-621 (1971).

1 Arthur D. Little Inc., "Stress Analysis of Ship-Suspended Heavily Loaded Cables for Deep Underwater Emplacements," Tech. Rep. No. 1370863 on Project Trident, Bureau of Ships Contr. NObsr-81564, Cambridge, Mass. (Aug. 1963).

${ }^{6}$ P. Holmes, "Mechanics of Raising and Lowering Heavy Loads in the Deep Ocean: Cable and Payload Dynamics," U. S. Naval Civil Eng. Lab. Tech. Rep. R-433 (Apr. 1966). 


\section{W. D. I WA N}

The effective mass of an element is assumed to include the actual air mass of that element plus any added mass resulting from hydrodynamic effects.

${ }^{7}$ W. D. Iwan, "A Generalization of the Concept of Equivalent Linearization," Dynamics Lab. Rep., California Inst. Technol., Pasadena, Calif. (Aug. 1971).

W. D. Iwan and E. J. Patula, "The Merit of Different Error Minimization Criteria in Approximate Analysis," J. Appl. Mech., Paper No. 70-092.
W. D. Iwan and I. M. Yang, "Application of Statistical Linearization Techniques to Nonlinear Multidegree-of-Freedom Systems," J. Appl. Mech., Paper No. 71-WA/APM-5.

10 E. T. Foster, Jr., "Model for Nonlinear Dynamics of Offshore Towers," J. Eng. Mech. Div. (Amer. Soc. Civil Eng.) 96, No. EMI, 41-67 (Feb. 1970).

11 W. J. Pierson, Jr., and L. Moskowitz, "A Proposed Spectral Form for Fully Developed Wind Seas Based on the Similarity Theory of S. A. Kitaigorodskii," J. Geophys. Res. 89, 5181-5190 (1964). 